\title{
Exploring a Nonlinear Collimation System for the LHC
}

\author{
R. Assmann, A. Faus-Golfe ${ }^{1)}$, S. Redaelli, J. Resta, G. Robert-Demolaize, D. Schulte, \\ F. Zimmermann \\ CERN, Geneva, Switzerland
}

\begin{abstract}
We explore the adaptation of a nonlinear collimation system, as previously developed for linear colliders, to LHC betatron cleaning. A possible nonlinear system for LHC consists of a horizontal and vertical primary collimator located in between a pair of skew sextupoles. We discuss the modified LHC optics, the need for and optimum placement of secondary absorbers, and the simulated cleaning efficiency.
\end{abstract}

${ }^{1)}$ University of Valencia, IFIC, Valencia, Spain

Presented at PAC 2005, Knoxville, USA, May 16-20, 2005

CERN,

CH-1211 Geneva 23,

Switzerland

Geneva, June 2005 


\title{
Exploring a Nonlinear Collimation System for the LHC
}

\author{
J. Resta, A. Faus-Golfe, IFIC, Valencia, Spain; \\ R. Assmann, S. Redaelli, G. Robert-Demolaize, D. Schulte, F. Zimmermann, CERN, Geneva
}

\section{Abstract}

We explore the adaptation of a nonlinear collimation system, as previously developed for linear colliders, to LHC betatron cleaning. A possible nonlinear system for LHC consists of a horizontal and vertical primary collimator located in between a pair of skew sextupoles. We discuss the modified LHC optics, the need for and optimum placement of secondary absorbers, and the simulated cleaning efficiency.

\section{INTRODUCTION}

A collimation system for the LHC should (i) prevent beam-loss induced quenches of the superconducting LHC magnets; (ii) minimize activation of accelerator components outside of the dedicated collimation insertions; (iii) ensure an acceptable background in the particle-physics experiments; (iv) withstand the impact of eight bunches in case of an irregular beam dump; and (v) not introduce intolerable wake fields that might compromise beam stability [1]. Larger aperture of the mechanical collimators is desired in order to avoid unacceptable high transverse resistive impedance from the collimators and to fulfil the above requirements. A possible solution could be the nonlinear collimation, described in the literature for linear colliders [2, 3, 4, 5].

For linear colliders designed to operate at center-of-mass energy $\sim \mathrm{TeV}$, the collimation requirements are similar to those for the LHC. It is thus a close thought to apply the same nonlinear collimation scheme as that designed for CLIC [2] (see Figure 1). The purpose of the first skew sextupole is to blow up beam sizes and particle amplitudes, so that the collimator jaw can be placed further away from the nominal beam orbit. A skew sextupole downstream of the spoiler, and at $\pi$ phase advance from the first sextupole, cancels the geometric aberrations induced by the former.

\section{SYSTEM LAYOUT}

In this section the optical constraints are derived for a system composed of a single spoiler and a pair of skew sextupole as illustrated in Figure 1.

The beam motion in a skew sextupole at a location with horizontal dispersion $D$ is given by the Hamiltonian

$$
H_{s}=\frac{1}{6} K_{s}\left(y^{3}-3(x+D \delta)^{2} y\right),
$$

where $x$ and $y$ are the transverse betatron amplitudes at the sextupole, and $\delta$ the relative momentum offset. The integrated sextupole strength $K_{s}$ can be expressed in terms

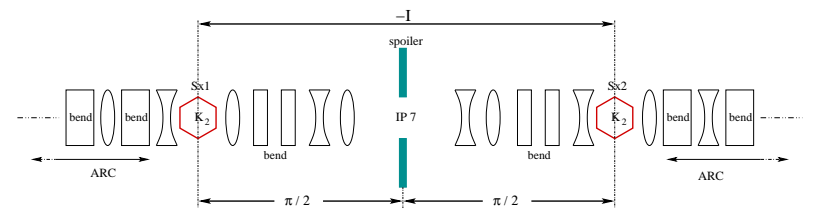

Figure 1: Schematic of a nonlinear collimation layout for the LHC.

of the sextupole length $l_{s}$, the pole-tip field $B_{T}$, the magnetic rigidity $(B \rho)$, and sextupole aperture $a_{s}$ as $K_{s}=$ $2 B_{T} l_{s} /(B \rho) a_{s}^{2}$.

At the skew sextupole a particle suffers deflections $\Delta x^{\prime}=-\partial H_{s} / \partial x, \Delta y^{\prime}=-\partial H_{s} / \partial y$ and the position at a downstream spoiler is obtained from

$$
\begin{aligned}
x_{\mathrm{sp}} & =x_{0, \mathrm{sp}}+R_{12} \Delta x^{\prime} \\
& \simeq K_{s} R_{12} x y \\
y_{\mathrm{sp}} & =y_{0, \mathrm{sp}}+R_{34} \Delta y^{\prime} \\
& \simeq-\frac{1}{2} K_{s} R_{34}\left(y^{2}-x^{2}\right) .
\end{aligned}
$$

where the subindex 0 indicates the position in the absence of the skew sextupole and $R_{12}, R_{34}$ are the optical transport matrix elements between the sextupole and the spoiler. In the second step we have neglected the dispersive term, since in the LHC dispersion times energy spread is small compared with the betatron amplitudes.

For spoiler survival in case of beam impact, a minimum beam size $\sigma_{r, \text { min }}$ of about $200 \mu \mathrm{m}$ is required, so that

$$
\sigma_{y} \sigma_{x} \geq \sigma_{r, \min }^{2}
$$

The rms beam size at the spoiler is computed by squaring the expressions for $x_{\mathrm{sp}}$ and $y_{\mathrm{sp}}$, and averaging over the transverse distribution. Detailed calculation can be found in Ref. [6]. The Eq. (4) determines the minimum values of $K_{s}, R_{12}$ and $R_{34}$ required.

We denote the collimation amplitude defined by the skew sextupole for the horizontal and vertical betatron motion as $\pm n_{x} \sigma_{x \text {,sext }}$ and $\pm n_{y} \sigma_{y \text {,sext }}$, respectively, and the physical aperture of the spoiler by $\pm n_{x 2} \sigma_{x, \mathrm{sp}}$ and $\pm n_{y 2} \sigma_{y, \mathrm{sp}}$.

For the collimation to function in either transverse plane, we must have [6]

$$
\begin{aligned}
& \frac{1}{2} K_{s} R_{34} n_{x}^{2} \beta_{x, \text { sext }} \epsilon_{x}=n_{y 2} \sqrt{\beta_{y, \mathrm{sp}} \epsilon_{y}} \\
& \frac{1}{2} K_{s} R_{34} n_{y}^{2} \beta_{y, \text { sext }} \epsilon_{y}=n_{y 2} \sqrt{\beta_{y, \mathrm{sp}} \epsilon_{y}} .
\end{aligned}
$$

Particles with $\left|y_{\text {sext }}\right| \approx\left|x_{\text {sext }}\right|$ will not be collimated by the vertical spoiler. To catch these particles as well, we 
use the horizontal deflection by the skew sextupole and the horizontal aperture of the spoiler, $n_{x 2} \sigma_{\mathrm{sp}}$. We require

$$
K_{s} R_{12} n_{x} n_{y} \sqrt{\beta_{x, \text { sext }} \beta_{y, \text { sext }}} \sqrt{\epsilon_{x} \epsilon_{y}}=n_{x 2} \sqrt{\beta_{x, \text { sp }} \epsilon_{x}}
$$

where the horizontal amplitude aperture at the spoiler $n_{x 2}$ can be adjusted to improve the cleaning efficiency for particles with offsets in both transverse planes. For example, we might set $n_{x 2}=2 n_{y 2}$, if the horizontal and vertical beta functions are equal at the skew sextupole and we want to approximate a circular collimation aperture in the transverse normalized $x-y$ space.

The tightest constraint likely arises from the achievable skew sextupole strength.

\section{OPTICS SOLUTION}

Let us take $n_{x}=n_{y} \approx 6$ and $n_{y 2}=8$ for a realistic collimation efficiency analysis. Fig. 2 shows the relation between the physical aperture of the spoiler $n_{x 2}$ and the collimation amplitude $n_{x}$ (defined by the sextupole) as given by Eq. (5).

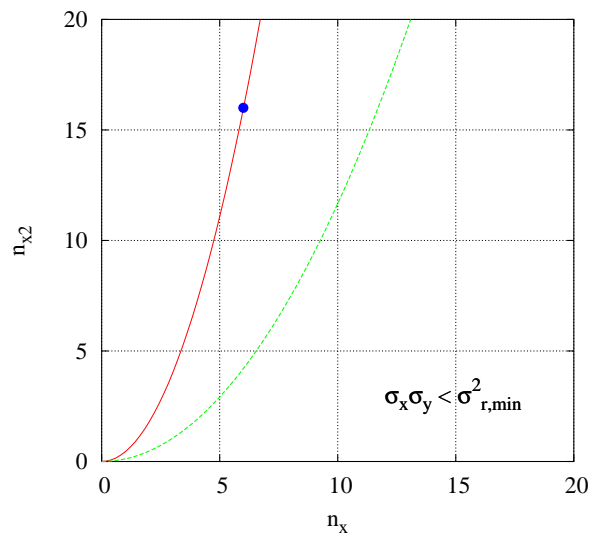

Figure 2: Physical spoiler aperture vs collimation amplitude defined by the skew sextupole. The solid line is the relation $n_{x 2}$ vs $n_{x}$ as given by Eq. (5). The dashed line represents the limit $\sigma_{x} \sigma_{y}=\sigma_{r \text {,min }}^{2}$ for spoiler survival when $n_{x 2}=2 n_{y 2}$. In this paper the point $n_{x}=6$ and $n_{x 2}=2 n_{y 2}=16$ is considered.

The optics for IR7 in LHC optics version V6.5 has been matched to fulfil the above equations and requirements, minimizing both the sextupole strength and the product of sextupole strength and beta function at the sextupole to reduce the nonlinear aberrations introduced by the first skew sextupole. This matching was done without affecting the optics of the other LHC insertions, and involved only existing quadrupole magnets [6]. Table 1 summarizes the main parameters of this optics including the strengh of the skew sextupoles. The corresponding plot for the beta functions is given in Figure 3.

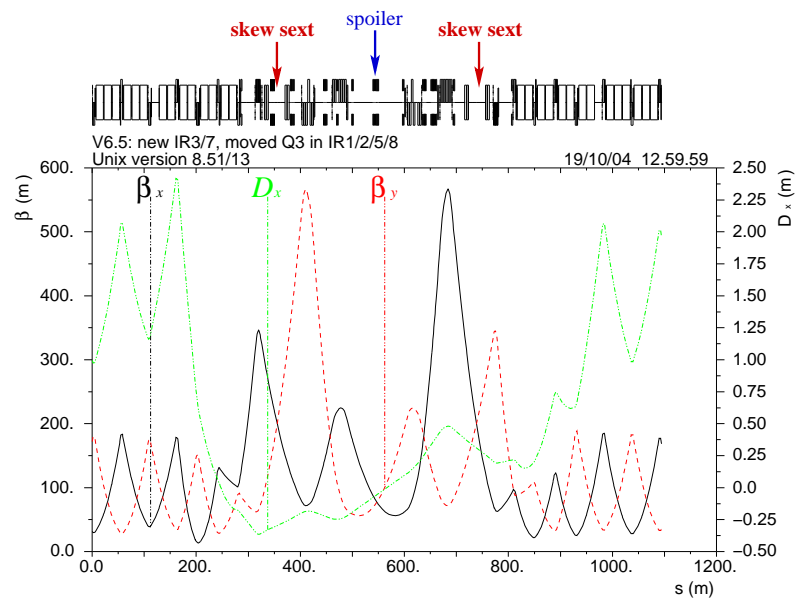

Figure 3: The optics solution proposed for LHC IR7 with a nonlinear collimation section based on two skew sextupoles.

Table 1: Optics parameters for a nonlinear collimation section in IR7 of LHC.

\begin{tabular}{lc}
\hline \hline variable & value \\
\hline beta functions $(x, y)$ at skew sext. & $200.0,200.0 \mathrm{~m}$ \\
product of skew sextupole pole-tip & \\
field and length $\left(B_{T} l_{s}\right)$ & $8.1823 \mathrm{~T} \cdot \mathrm{m}$ \\
skew sextupole aperture $a_{s}$ & $10 \mathrm{~mm}$ \\
skew sextupole strength $K_{s}$ & $7.0063 \mathrm{~m}^{-2}$ \\
$R_{12}, R_{34}$ from sext. to spoiler & $124.403,124.404 \mathrm{~m}$ \\
beta functions $(x, y)$ at spoiler & $77.381,77.381 \mathrm{~m}$ \\
rms spot size $(x, y)$ at spoiler & $215.89,263.96 \mu \mathrm{m}$ \\
\hline
\end{tabular}

\section{CLEANING EFFICIENCY}

The lattice of Figure 3 was adapted to thin-lens in order to use the thin-lens version of the tracking program Sixtrack [7]. This tool allows us to calculate the cleaning inefficiency of the collimation system and to save the particle trajectories for an offline analysis of beam losses.

In a first step, the optics solution from the previous section has been implemented with all the available collimators from the official LHC collimation system design [1]. The goal is to see how the official system (linear optics) could be improved by the skew sextupoles. Figure 4 compares the trajectory of a halo particle in the case of linear and nonlinear optics. For the nonlinear optics, the first sextupole gives a kick which increases the transversal amplitude in the region where the collimators are located. The second sextupole cancels the effect induced by the former.

The cleaning inefficiency $\eta_{c}\left(A_{0}\right)$ of the collimation system is defined [1] as a function of the particle amplitude $A_{0}$ as the number of beam protons with amplitude above $A_{0}, N_{p}\left(A>A_{0}\right)$, divided by the total number of absorbed protons in the cleaning insertion, $N_{\text {abs }}$ : 


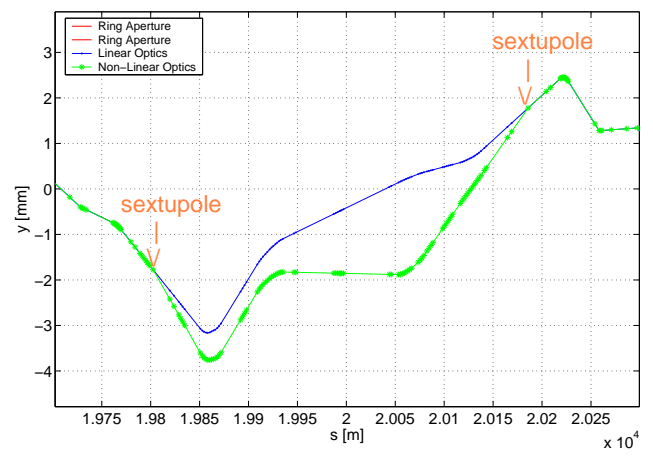

Figure 4: trajectory of a halo particle in LHC IR7 for the case of the linear optics (blue curve) and the optics with the sextupole pair (green curve).

$$
\eta_{c}\left(A_{0}\right)=\frac{N_{p}\left(A>A_{0}\right)}{N_{\mathrm{abs}}} .
$$

$N_{p}=50240$ protons have been tracked for 200 turns. Figure 5 shows the cleaning inefficiency at $7 \mathrm{TeV}$ for different apertures of the secondary collimators. The cleaning efficiency is $\eta_{c} \approx 0.007$ at a radial amplitude of $10 \sigma$, while that at the same radial amplitude is $\eta_{c} \approx 0.0003$ for the conventional collimation system. Figure 6 shows that a maximum absorption is found in the collimator S.B4L7, located near IP7, for an aperture of the secondary collimators equal to $9 \sigma$.

Although the nonlinear collimation could help reducing the collimator impedances, the cleaning efficiency of the present configuration is a factor 20 worse than for the conventional collimation system. However, an optimization of the nonlinear system has not yet been performed.

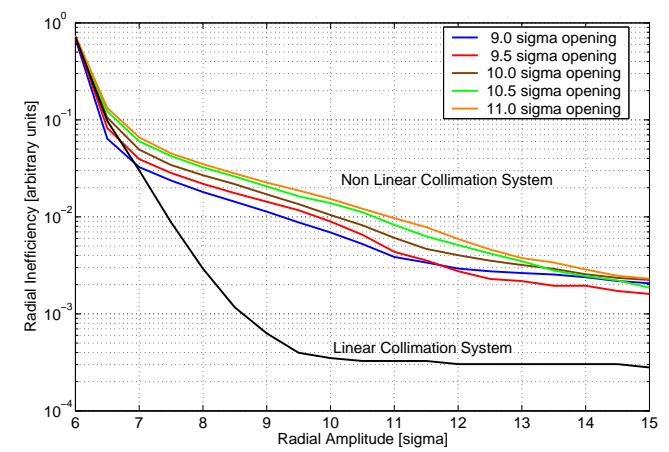

Figure 5: Cleaning inefficiency, $\eta_{c}\left(A_{0}\right)$, as a function of the radial amplitude $A_{0}$. The case for the nonlinear system is plotted for different apertures of the secondary collimators, and compared with the case of the conventional linear collimation system (black line).

\section{CONCLUSIONS}

The viability of a nonlinear collimation system for the LHC based on 1 pair of skew sextupoles has been explored.

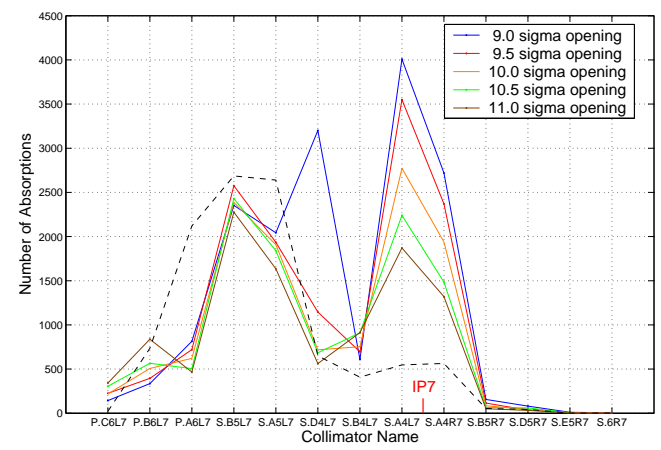

Figure 6: Number of particles absorptions in the collimators of the IR7 of the LHC. The following apertures for the secondary collimators are considered: $9 \sigma, 9.5 \sigma, 10.0 \sigma$, $10.5 \sigma$ and $11 \sigma$. The black dashed line corresponds to the absorption for the linear system.

Assuming basic requirements and criteria, such as the minimum beam size $\left(\sigma_{r, \min } \approx 200 \mu \mathrm{m}\right)$ for the spoiler survival in case of beam impact, the optics parameters for the nonlinear lattice were calculated.

The first tracking studies for the nonlinear system have shown a value for the cleaning inefficiency approximately 20 times higher than that of the conventional collimation system. It is worthwhile to mention that the collimator gap settings between the sextupoles were not fully optimized, but that all collimators were retracted by the same amplitude in units of linear sigma.

In this paper the principal functioning of the nonlinear collimation concept has been checked for the LHC. Future tracking studies should be performed with a single spoiler at IP7 and secondary collimators placed at the optimum locations behind the spoiler.

\section{REFERENCES}

[1] R. Assmann et al., "Collimators and Cleaning, Could this Limit the LHC Performance?," LHC Project Workshop'Chamonix XII' (2003).

[2] A. Faus-Golfe, F. Zimmermann, "A Nonlinear Collimation System for CLIC,” EPAC 2002 Paris (2002).

[3] L. Merminga et al., "Collimation systems for a TeV linear collider,” Part. Accel. 48, 85 (1994); SLAC-PUB-5165 Rev. May 1994.

[4] R. Brinkmann, P. Raimondi and A. Seryi, "Halo reduction By Means of Non Linear Optical Elements in the NLC Final Focus System," PAC2001, Chicago (2001).

[5] R. Brinkmann, N. J. Walker and G. A. Blair, "The TESLA Post-linac Collimation System," DESY TESLA-0112 (2001).

[6] A. Faus-Golfe, J. Resta López, F. Zimmerman, Proceedings of HHH-2004; see web site: http://carehhh.web.cern.ch/CARE-HHH/HHH-2004.

[7] G. Ripken and F. Schmidt, CERN SL 95-12 (AP)(1995), DESY 95-063 (1995). 